\title{
Are There Regional Variations in the Diagnosis, SuRVEILlaNCE, AND CONTROL OF METHICILlin- RESISTANT STAPHYLOCOCCUS AUREUS?
}

\author{
Hervé M. Richet, MD; Mohamed Benbachir, PhD; Derek F. J. Brown, PhD; Helen Giamarellou, MD; Ian Gould, MD; Marija Gubina, MD; \\ Piotr Heczko, MD; Smilja Kalenic, MD; Marina Pana, MD; Didier Pittet, MD; Saida Ben Redjeb, MD; Jiri Schindler, MD; \\ Carlos Starling, MD; Marc J. Struelens, MD; Wolfgang Witte, MD; William R. Jarvis, MD; International Network for the Study and \\ Prevention of Emerging Antimicrobial Resistance (INSPEAR)*
}

\begin{abstract}
OBJECTIVE: To assess the way healthcare facilities (HCFs) diagnose, survey, and control methicillin-resistant Staphylococcus aureus (MRSA).

DESIGN: Questionnaire.

SETTING: Ninety HCFs in 30 countries.

RESULTS: Evaluation of susceptibility testing methods showed that 8 laboratories (9\%) used oxacillin disks with antimicrobial content different from the one recommended, $12(13 \%)$ did not determine MRSA susceptibility to vancomycin, and 4 (4.5\%) reported instances of isolation of vancomycin-resistant $S$. aureus but neither confirmed this resistance nor alerted public health authorities. A MRSA control program was reported by 55 (61.1\%) of the HCFs. The following isolation precautions were routinely used: hospitalization in a private room (34.4\%), wearing
\end{abstract}

of gloves $(62.2 \%)$, wearing of gowns $(44.4 \%)$, hand washing by healthcare workers (53.3\%), use of an isolation sign on the patient's door (43\%), or all four. When the characteristics of HCFs with low incidence rates $(<0.4$ per 1,000 patient-days) were compared with those of HCFs with high incidence rates $(\geqslant 0.4$ per 1,000 patient-days), having a higher mean number of beds per infection control nurse was the only factor significantly associated with HCFs with high incidence rates (834 vs 318 beds; $P=$ $.02)$.

CONCLUSION: Our results emphasize the urgent need to strengthen the microbiologic and epidemiologic capacities of HCFs worldwide to prevent MRSA transmission and to prepare them to address the possible emergence of vancomycin-resistant S. aureus (Infect Control Hosp Epidemiol 2003;24:334-341).

Drs. Richet and Jarvis are from the Investigation and Prevention Branch, Hospital Infections Program, Centers for Disease Control and Prevention, Atlanta, Georgia. Dr. Benbachir is from the Laboratoire de Microbiologie, Centre Hospitalier Ibn Rochd, Casablanca, Maroc. Dr. Brown is from the Clinical Microbiology and Public Health Laboratory, Addenbrooke's Hospital, Cambridge, United Kingdom. Dr. Giamarellou is from the 4th Department of Internal Medicine, Sismanoglio General Hospital, Maroussi Attikis, Greece. Dr. Gould is from Clinical Microbiology, Aberdeen Royal Hospitals, NHS Trust, Aberdeen, Scotland, United Kingdom. Dr. Gubina is from the Institute of Microbiology and Immunology, Medical Faculty, University of Ljubljana, Ljubljana, Slovenia. Dr. Heczko is from the Microbiology Department, Jagiellonian University Medical School, Krakow, Poland. Dr. Kalenic is from the Department of Clinical and Molecular Microbiology, Zagreb University School of Medicine, Zagreb, Croatia. Dr. Pana is from the Streptococcus National Reference Center, Institutul Cantacuzino, Bucarest, Romania. Dr. Pittet is from the Unité de Prévention et de Contrôle de l'Infection, Hôpitaux Universitaires de Genève, Genève, Suisse. Dr. Redjeb is from the Laboratoire de Bactériologie, Hôpital Charles Nicolle, Tunis, Tunisie. Dr. Schindler is from the Department of Medical Microbiology, 3rd Faculty of Medicine, Charles University, Prague, Czech Republic. Dr. Starling is from Felicio Rocho, Vera Cruz and São Francisco Hospitals, Belo Horizonte, Brazil. Dr. Struelens is from the Department of Microbiology, Université Libre de Bruxelles, Hôpital Erasme, Bruxelles, Belgique. Dr. Witte is from the Robert-Koch Institüt, Bunderinstitüt für Infektionskrankheiten, Wernigerode, Germany.

Address reprint requests to Hervé M. Richet, MD, Laboratoire de Bactériologie-Virologie-Hygiène Hospitalière, Institut de Biologie des Hôpitaux de Nantes, 9, quai Moncousu, BP 1005, 44093 Nantes cedex 01, France.

${ }^{*}$ INSPEAR MRSA survey participants-Argentina: Dr. Horacio Lopardo (Buenos Aires); Austria: Prof. Manfred Rotter (Vienna); Belgium: Prof. Marc Struelens (Bruxelles); Bosnia-Herzegovina: Dr. Maja Ostjic; Brazil: Dr. Jorge Saliba, Dr. Carlos Starling (Belo Horizonte, Minas Gerais); Bulgaria: Prof. Boyka Markova (Sofia); China: Prof. Kwok-Yung Yuen (Hong Kong); Côte d'Ivoire: Prof. Mireille Dosso (Abidjan); Croatia: Dr. Ines Bencic, Prof. Smilja Kalenic, Dr. Nastja Kucisec-Tepes, Dr. Jasenta Skrlin (Zagreb), Dr. Edita Susic (Sibenik), Dr. Maja Tomic (Slavonski Brod); Czech Republic: Dr. D. Burgetova (Brno), Dr. Jini Schindler (Prague); France: B. Andre-Richet (Nantes), D. Barraud (Gonesse), Dr. Besnard (Mayenne), Dr. Edouard Bichier (Saumur), Mme. M. Bingen (Gonesse), Dr. Catherine Branger (Clichy), Mme. Anne Cirioni, Mme. Stephanie Clavel (Nogent le Rotrou), Dr. Simone Clévenot (Pithiviers), Dr. Andréa Collet (Port-Luois), Dr. François Coulomb (Dreux), Prof. Patrice Courvalin (Paris), Mme. Armelle David (Saint Gemmes), Prof. François Denis Dr. Marie Cécile Ploy (Limoges), Dr. Jean Yves Esvant (Paimpol), Dr. Ferrant (Lamembrolle sur choisille), Mme. Annick Genuis (La Ferté Bernard), Dr. Guir (Paimbeuf), Dr. Didier Jan (Mayenne), Mme. Françoise Jezequel (Roscoff), Dr. Jean Louis Laborie (Lannion), Dr. Florence Le Gallou (Nantes), Dr. Lemoign (Bayeux), Dr. Christine Rennes (Saint Lo), Dr. Pascale Richard (Morlaix), Mme. Yveline Ruellan (Vannes), Dr. Jacques Vaucel (Saint Brieux), Dr Liliane Zbierski (Laval); Germany: Dr. Barbel Christiansen (Kiel), Dr. Julia Fitzner (Berlin), Dr. Mathias Hermann (Muenster), Dr. Michael Kramer (Bonn), Dr. Werner Mathys (Muenster), Dr. Sabine Schubert (Kiel), Dr Stefan Weber (Hanover), Prof. Wolfgang Witte (Wernigerode); Greece: Prof. Helen Giamarellou (Maroussi Attikis), Dr. A. Katrachoura (Piraeus), Dr. E. A. Papafrangas (Maroussi Attikis), Dr. Danae Sofianou (Thessaloniki); Hungary: Dr. Anna Marton (Budapest); Israel: Dr. Nathan Keller, Prof. Ethan Rubinstein (Tel Hashomer); Italy: Dr. Giuseppe Cornaglia (Verona); Latvia: Dr.Juriis Perevostikos, Dr. Ivonna Selga (Riga); Luxembourg: Dr Robert Hemmer (Luxembourg); Morocco: Prof. M. A. Alaoui, Prof. A. Benouda-Benjilaji (Rabat), Prof. Mohamed Benbachir (Casablanca); Netherlands: Dr. J. H. T. Wagenvoort (Heerlen); Poland: Prof. Piotr Heczko, Dr. M. Pytlos (Cracaw), Prof. Waleria Hryniewicz (Warsaw); Romania: Dr. Manuela Anda Andrei, Dr. Dana Andries, Dr. Daniela Blana, Prof. Lucia Debeleac, Dr. Olga Dorobat, Dr. Antonia Medvetchi-Panatescu, Dr. Irina Nistor, Dr. Maria Pana, Dr. Raluca Papagheorge, Dr. Elena Silaghi, Dr. Daniela Tudorache (Bucuresti); Russia: Dr. Andrei Dekhitch, Prof. Leonid Stratchounski (Smolensk), Senegal: Prof. S. B. Cheikh Boye (Dakar); Slovenia: Prof. Marija Gubina, Dr. Katja Seme (Ljubljana); Spain: Dr. Javier Garau, Dr. Ferran Navarro, Prof. Guillem Prats, Dr. Antoni Trilla (Barcelona), Prof. Rafael Gomez-Lus (Zaragoza); Switzerland: Prof. Raymond Auckenthaler, Prof. Didier Pittet (Geneva), Dr. Enos Bernasconi, Prof. Jean Claude Piffaretti (Lugano), Dr. Reno Frei, Dr. Andreas Widmer (Basel); Tunisia: Prof. Assia Ben Hassen, Dr. Amel Kechrid, Prof. Saida Ben Redjob (Tunis); United Kingdom: Dr. Derek Brown, Dr. Nick Brown (Cambridge), Dr. Adam Fraise (Birmingham), Dr. Ian Gould (Aberdeen); United States: Dr. Krishna Agarwal, Dr. David Pegues (Los Angeles), Dr. Steven Gordon (Cleveland), Dr. William Jarvis, Dr. Hervé Richet, Dr. Fred Tenover (Atlanta), Dr. Leonard Mermel (Providence), Dr. Louis Polish (Saint Louis).

The authors thank Béatrice André-Richet, PharmD, MPH, and Elaine Miller, RN, MPH, for their assistance with data entry and analysis. 
The emergence of resistance to antimicrobial agents has become a major public health problem worldwide in both community and healthcare settings. ${ }^{1-13}$ Emergence and spread of multidrug-resistant pathogens is facilitated when laboratory personnel have difficulty accurately detecting these organisms, the medical community does not identify and isolate patients colonized or infected with these organisms, and infection control interventions are either not implemented, ineffective, or implemented so late that the organisms have become endemic. ${ }^{7,-11}$ The global spread of methicillin-resistant Staphylococcus aureus (MRSA) demonstrates these issues. MRSA emerged in Europe 40 years ago, concomitant with the introduction of methicillin. During the mid-1980s, epidemic strains spread in hospitals throughout the world and in most hospitals few, if any, infection control precautions were implemented until these strains had become endemic. ${ }^{14-16}$ Currently, the large reservoir of patients colonized or infected with MRSA, many of whom are unrecognized, interferes with effective infection control. ${ }^{17}$

Recently, infections caused by MRSA strains with reduced susceptibility to glycopeptides have been reported from Japan, the United States, and France, signaling the potential emergence of $S$. aureus strains with full resistance to vancomycin. ${ }^{13,18,19}$ In addition, the recent emergence of community-acquired MRSA infections in Australia, Canada, France, and the United States will further complicate efforts to control MRSA. ${ }^{20-23}$ The report of four pediatric deaths due to community-acquired MRSA demonstrates the potential severity of these infections. ${ }^{21}$

Because of the continued emergence of multidrugresistant $S$. aureus, we considered it critical to assess the methods currently used by hospitals worldwide to control the emergence of MRSA. The objectives of this survey were to assess (1) the methods used by laboratories to identify $S$. aureus and determine the susceptibility of this pathogen to antimicrobials, including methicillin; (2) the MRSA surveillance and control programs in these hospitals; and (3) the incidence of MRSA infections in hospitals worldwide.

\section{METHODS}

The International Network for the Study and Prevention of Emerging Antimicrobial Resistance (INSPEAR) is a consortium of clinical microbiologists, hospital epidemiologists, infectious disease specialists, experts in the fields of antimicrobial resistance, public health agencies, and national reference laboratories whose purpose is to serve as an early warning system for emerging antimicrobial resistance.

In May 1998, a questionnaire was sent to all INSPEAR centers to assess the way they currently identify, survey, and control MRSA. The form included questions about hospital characteristics, laboratory methods used to identify staphylococci and perform antimicrobial susceptibility testing, and the existence of MRSA surveillance and control programs. Institutions were also asked to provide for each year, from 1990 to 1997, the total num- ber of admissions, patient-days of hospitalization, $S$. aureus and MRSA isolates, and MRSA isolates from blood cultures. If these data were not available annually during this period, the centers were asked to provide data for 1997 only. Laboratorians were asked to report only one isolate per patient and to exclude isolates obtained by colonization screening.

Because the number of healthcare facilities by country varied from 1 to 21 , it was impossible to stratify the results of the survey by country and the centers were grouped into 5 regions: Africa $(\mathrm{N}=7)$, Eastern Europe ( $N$ $=27$ ), Western Europe $(N=43)$, South America $(N=6)$, and the United States $(N=5)$. The Middle East and Asia with one center each were not included in the stratified analysis.

Data were analyzed with Epi-Info software (version 6; Centers for Disease Control and Prevention, Atlanta, GA).

Risk factors for having a high rate of MRSA infections in 1997 were assessed by comparing the characteristics (country, type of hospital [teaching, public, or private], number of beds, presence and type of MRSA surveillance and control programs, and number of infection control nurses) of healthcare facilities with high incidence rates (those with MRSA infection rates per patient-days of 0.4 or greater, which was the median infection rate in 1997) with the characteristics of healthcare facilities with low incidence rates (MRSA infection rates per patientdays of less than 0.4).

Univariate analyses were performed using either the chi-square or Fisher's exact test for proportions and the Student's $t$ test for means.

\section{RESULTS}

Of 110 INSPEAR healthcare facilities in 33 countries, 90 healthcare facilities ( $81.8 \%)$ from 30 countries participated in this survey.

\section{Hospital Characteristics}

Most of the 90 participating healthcare facilities were public hospitals $(84.4 \%$ ) or teaching hospitals (72.2\%) with a median of 640 beds (range, 50 to 3,889 ) admitting a median of 19,790 patients (range, 342 to $98,298)$ in 1997. Most of the healthcare facilities had adult intensive care units (84\%) and medical services (93.5\%) and surgical services (93.5\%). Fewer than half of the healthcare facilities had either a neonatal intensive care unit $(44.4 \%)$ or a pediatric intensive care unit $(42 \%)$, or a long-term-care facility (36.7\%).

\section{Methods Used to Identify Staphylococci}

Laboratory personnel used a combination of different methods to identify $S$. aureus, including catalase $(87.8 \%)$, tube coagulase $(68.9 \%)$, in-house agglutination (25.6\%), and commercial agglutination $(68.9 \%)$. When needed, they performed biochemical identification (74.4\%).

\section{Methods Used for Susceptibility Testing}

For antimicrobial susceptibility testing, the diskdiffusion method was most commonly used (79 of 90 , 
$88 \%$ ), followed by automated methods ( 26 of $90,29 \%$ ). Sixty-one (68\%) of the laboratories used only the disk-diffusion method, 8 (9\%) used only an automated method, and $18(20 \%)$ used both automated and disk-diffusion methods.

Minimum inhibitory concentrations were determined by $38(42.2 \%)$ of the laboratories for selected antimicrobials or in selected circumstances; the Etest (AB Biodisk, Solna, Sweden) was the most common technique used ( 35 of $38,92.1 \%$ ).

Automated methods were used mostly by Western European laboratories (17 of 26, 65.4\%), whereas all laboratories in Africa, Eastern Europe, and South America used only the disk-diffusion method.

Four different interpretative breakpoints were used by the laboratories: the British Society for Antimicrobial Chemotherapy breakpoints were used by 6 laboratories (6.7\%); the Comité de l'Antibiogramme de la Société Française de Microbiologie (France) breakpoints were used by 26 laboratories (28.9\%); the Deutsches Institute für Normung (Germany) breakpoints were used by 4 laboratories (4.4\%); and the National Committee for Clinical Laboratory Standards (United States) breakpoints were used by 55 laboratories (61.1\%). Four laboratories (4.4\%) used breakpoints from both the Comite de l'Antibiogramme de la Société Française de Microbiologie and the National Committee for Clinical Laboratory Standards. To detect methicillin resistance among $S$. aureus, 48 (53\%) of the laboratories used 1- $\mu g$ oxacillin disks and $35(38.9 \%)$ used 5- $\mu$ g disks. A comparison between the type of oxacillin disk used and the recommended breakpoints illustrates the problem caused by the multiplicity of interpretative breakpoints. The Comite de l'Antibiogramme de la Société Française de Microbiologie recommends using 5 - $\mu \mathrm{g}$ oxacillin disks; however, one center supposedly following these recommendations used a 1ug disk. On the other hand, although the National Committee for Clinical Laboratory Standards recommends using a 1- $\mu$ g oxacillin disk, 7 centers (12.7\%) that reported using these recommendations used 5-ug disks.

The reading of the disk-diffusion inhibition zone to assess whether a strain was resistant to oxacillin varied by laboratory. Sixty $(66.7 \%)$ determined resistance by measuring the inhibition zone diameter, $56(62.2 \%)$ by looking for the presence of small colonies of staphylococci (squatter colonies) within the inhibition zone, and 40 (44.4\%) by measuring the inhibition zone and looking for squatter colonies. In addition, 30 (33.7\%) of the laboratories used screening plates containing $6 \mu \mathrm{g} / \mathrm{mL}$ of oxacillin and $\mathrm{NaCl}$ to detect MRSA. This was performed in combination with the disk-diffusion method in 23 laboratories $(25.6 \%)$, with the automated method in 1 laboratory, and with both disk and automated methods in 6 laboratories (6.7\%).

\section{Laboratory Quality Assurance Programs for Antimicrobial Susceptibility Testing}

Seventy-eight laboratorians (86.7\%) reported the presence of a quality assurance program; $65(72.2 \%)$ were internal programs and 48 (53.3\%) were external. Only 38 laboratorians $(42.2 \%)$ participated in both programs.

\section{Antimicrobial Resistance}

Seventy-eight $(87 \%)$ of the laboratorians tested MRSA susceptibility to vancomycin and $40(44 \%)$ tested MRSA susceptibility to teicoplanin. Four of the laboratorians reported rates of MRSA resistance to vancomycin of $1.4 \%, 3.7 \%, 5.7 \%$, and $25 \%$, respectively, and one laboratorian reported a rate of resistance to teicoplanin of $6 \%$. Unfortunately, these laboratorians did not save their isolates, send them to reference laboratories for confirmation, or alert public health authorities to the existence of their strains. Thus, we were unable to confirm the accuracy of these reports.

\section{Presence of Infection Control Nurses}

Fifty-one healthcare facilities (56.7\%) reported the presence of at least one infection control nurse, with a median infection control nurse-to-bed ratio of 1 infection control nurse for 450 beds (range, 1:50 to 1:3,334). The highest infection control nurse-to-bed ratio was observed in South American healthcare facilities (median, 1:191; range, $1: 80$ to 1:324), followed by Western European healthcare facilities (median, 1:503; range, 1:130 to $1: 3,334$ ) and Eastern European healthcare facilities (median, 1:615; range, $1: 52$ to $1: 1,104)$.

\section{Surveillance Activities}

The existence of a MRSA surveillance program was reported by $59(65.6 \%)$ of the healthcare facilities. The most common surveillance method used was the calculation of the proportion of all $S$. aureus isolates resistant to methicillin ( 45 of $90,50 \%$ ), followed by the calculation of MRSA incidence rate per admission (30 of $90,33.3 \%$ ) or per patient-days of hospitalization ( 20 of $90,22.2 \%$ ). Rates were calculated for the entire healthcare facility by $81.4 \%$ ( 48 of 59) of the healthcare facilities performing surveillance and separately for each service by $52.5 \%$ (31 of 59). The surveillance rates were reported to clinicians on each service every month in $20.3 \%$ (12 of 59 ) of the healthcare facilities, every 3 months in 17\% (10 of 59), every 6 months in $39 \%$ ( 23 of 59 ), and yearly in $25.4 \%$. Eighty-eight percent (52 of 59) of the centers used the Centers for Disease Control and Prevention definitions of nosocomial infections. The year of implementation of MRSA rate calculations and the denominators used varied by healthcare facility (Figure). During the 1980 s, few hospitals conducted surveillance for MRSA. Subsequently, the proportion of hospitals conducting MRSA surveillance steadily increased in the early 1990 s, reaching $65 \%$ by 1997 (Figure).

In 1997, Western Europe (36 of $43,83.7 \%$ ) had the highest proportion of healthcare facilities reporting the existence of a MRSA surveillance program and calculating the proportion of $S$. aureus that were methicillin resistant (25 of $43,58 \%$ ), the MRSA incidence per admission (24 of $43,56 \%$ ), and the MRSA incidence per patient-days 
TABLE 1

Methicillin-Resistant Staphrlococcus aureus Surveillance Programs and Methods by Region, INSPEAR, 1997

\begin{tabular}{lccrr}
\hline Continent & $\begin{array}{c}\text { Survelliance } \\
\text { Program }\end{array}$ & $\begin{array}{c}\text { Proportion of } \\
\text { S. aureus Methicillin Resistant }\end{array}$ & $\begin{array}{c}\text { Incidence per } \\
\text { Admission }\end{array}$ & $\begin{array}{r}\text { Incidence per } \\
\text { 1,000 Patient-Days }\end{array}$ \\
\hline Africa & $3 / 6(50 \%)$ & $1 / 6(17 \%)$ & $1 / 6(17 \%)$ & $0 / 6$ \\
Eastern Europe & $13 / 27(48 \%)$ & $13 / 27(48 \%)$ & $3 / 27(11 \%)$ & $3 / 27(11 \%)$ \\
Western Europe & $36 / 43(84 \%)$ & $25 / 43(58 \%)$ & $24 / 43(56 \%)$ & $15 / 43(35 \%)$ \\
South America & $4 / 6(67 \%)$ & $4 / 6(67 \%)$ & $2 / 6(33 \%)$ & $2 / 6(33 \%)$ \\
United States & $2 / 5(40 \%)$ & $1 / 5(20 \%)$ & $0 / 5$ & $0 / 5$ \\
& & & & \\
\hline
\end{tabular}

INSPEAR - International Network for the Study and Prevention of Emerging Antimicrobial Resistance.

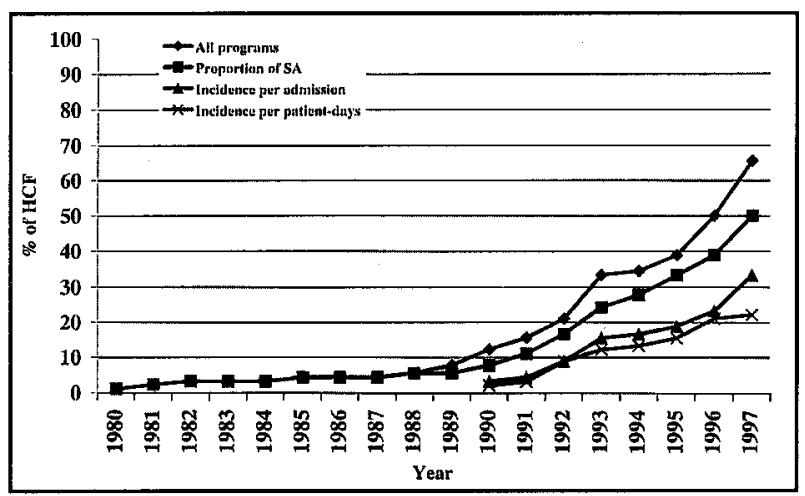

FIGURE. Year of implementation of methicillin-resistant Staphylococcus aureus (MRSA) rate calculations and denominator used (percentage of healthcare facilities [HCFs]). $S A=S$. aureus.

(15 of $43,35 \%)$. In contrast, only $1(16.7 \%)$ of 6 African healthcare facilities, 3 (11\%) of 27 Eastern European healthcare facilities, 2 (33\%) of 6 South American healthcare facilities, and none of the U.S. healthcare facilities calculated the incidence per admissions. Fewer healthcare facilities in each region calculated the incidence per patients-days, the highest proportion being observed among Western European healthcare facilities (15 of 43, 35\%) (Table 1).

\section{Surveillance Results}

A total of 12,828 MRSA strains (median, 102; range, 1 to 1,196$)$ were isolated at participating laboratories in 1997 . For this year, we were able to calculate the proportion of $S$. aureus that were resistant to methicillin for $64.4 \%$ of the healthcare facilities, the MRSA incidence rate per admission for $60 \%$, the incidence of MRSA bacteremia per admission for $38.9 \%$, and the MRSA incidence rate per patient-days for $34.4 \%$. Of the 51 healthcare facilities providing data used to calculate the following rates, 46 (90.2\%) had a quality assurance program; 41 (80.4\%) had an internal program and 24 (47\%) participated in an external program. The median proportion of $S$. aureus resistant to methicillin was $27.6 \%$ (range, $0 \%$ to $75 \%$ ); the median incidence rate of MRSA infections was 0.49 per 100 admissions (range, $0.02 \%$ to $8.8 \%$ ) and 0.43 per 1,000 patientdays of hospitalization (range, $0.04 \%$ to $3.7 \%$ ). The median
TABLE 2

INCIDENCE OF METHICILLIN-RESISTANT STAPHYLOCOCCUS AUREUS INFECTIONS PER 1,000 PATIENT-DAYS OF HOSPITALIZATION ACCORDING TO REgION AND HOSPITAL CATEgORY

\begin{tabular}{lll}
\hline & Medlan Incidence & Range \\
\hline Region & & \\
South America & 0.5 & $0.4-0.5$ \\
Eastern Europe & 0.5 & $0.06-2.4$ \\
Western Europe & 0.4 & $0-1.6$ \\
Hospital category & & \\
$0-499$ beds & 0.4 & $0.25-1$ \\
$500-999$ beds & 0.7 & $0.02-4$ \\
$\geqslant 1,000$ beds & 0.27 & $0-2.8$ \\
\hline
\end{tabular}

incidence of bacteremia caused by MRSA was 0.073 per 100 admissions (range, $0 \%$ to $16 \%$ ). When we used the most appropriate indicator for inter-hospital MRSA rate comparison (ie, the incidence per patient-days of hospitalization), the median incidence rates were similar by region and by hospital size. However, the MRSA infection rates varied widely within each region or within each hospital category (Table 2).

\section{MRSA Control Program}

In $1997,61.1 \%$ (55 of 90 ) of the healthcare facilities reported the existence of a MRSA control program, ranging from $81.5 \%$ (35 of 43 ) in Western Europe to $17 \%$ (1 of 6) in Africa.

The assessment of the type of isolation precautions implemented revealed that only $31(34.4 \%)$ of the healthcare facilities systematically hospitalized MRSA-infected patients in a private room; workers in the healthcare facilities wore gloves $(56,62.2 \%)$, gowns $(40,44.4 \%)$, or both during the care of MRSA-infected patients; hand washing was performed routinely in the patient's room in 48 (53.3\%) of the healthcare facilities; and an isolation sign was routinely posted on the patient's door in 39 (43\%) of the healthcare facilities (Table 3).

Wide variations in the use of isolation precautions were observed between regions. The proportion of hospitalized MRSA-infected patients placed in a private room ranged from $0 \%$ ( 0 of 6) in South America to $53.5 \%$ (23 of 
TABLE 3

Assessment of Isolation Precautions Used for Patients Infected With Methicillin-Resistant $S$ taphrlococcus aureus, INSPEAR, 1997

\begin{tabular}{|c|c|c|c|c|c|c|}
\hline & $\begin{array}{c}\text { All Centers } \\
(\mathbf{N}=\mathbf{9 0})\end{array}$ & $\begin{array}{l}\text { Africa } \\
(\mathrm{N}=6)\end{array}$ & $\begin{array}{c}\text { Eastern Europe } \\
(\mathbf{N}=\mathbf{2 7})\end{array}$ & $\begin{array}{c}\text { Western Europe } \\
(\mathbf{N}=\mathbf{4 3})\end{array}$ & $\begin{array}{l}\text { South America } \\
\qquad(\mathrm{N}=\mathbf{6}) \\
\end{array}$ & $\begin{array}{c}\text { United States } \\
(\mathbf{N}=\mathbf{5}) \\
\end{array}$ \\
\hline \multicolumn{7}{|l|}{ Private room } \\
\hline Routinely & $31(34.4 \%)$ & $1(17 \%)$ & $5(18.5 \%)$ & $23(53.5 \%)$ & 0 & $2(40 \%)$ \\
\hline Occasionally & $30(33.3 \%)$ & 0 & $8(29.6 \%)$ & $17(39.5 \%)$ & $3(50 \%)$ & 0 \\
\hline \multicolumn{7}{|l|}{ Glove use } \\
\hline Routinely & $56(62.2 \%)$ & $1(17 \%)$ & $11(40.7 \%)$ & $35(81.4 \%)$ & $2(33 \%)$ & $5(100 \%)$ \\
\hline Occasionally & $4(4.4 \%)$ & 0 & $1(3.7 \%)$ & $3(7 \%)$ & 0 & 0 \\
\hline \multicolumn{7}{|l|}{ Gown use } \\
\hline Routinely & $40(44.4 \%)$ & 0 & $7(26 \%)$ & $28(65 \%)$ & $2(33 \%)$ & $3(60 \%)$ \\
\hline Occasionally & $15(16.7 \%)$ & 0 & $3(11 \%)$ & $10(23.3 \%)$ & $1(17 \%)$ & 0 \\
\hline \multicolumn{7}{|c|}{ Hand washing in patient room } \\
\hline Routinely & $48(53.3 \%)$ & $1(17 \%)$ & $11(40.7 \%)$ & $27(62.8 \%)$ & $4(67 \%)$ & $3(60 \%)$ \\
\hline Occasionally & $7(8 \%)$ & 0 & $2(7.4 \%)$ & $5(11.6 \%)$ & 0 & 0 \\
\hline \multicolumn{7}{|c|}{ Isolation sign on patient's door } \\
\hline Routinely & $39(43 \%)$ & 0 & $8(29.6 \%)$ & $25(58 \%)$ & $4(67 \%)$ & $2(40 \%)$ \\
\hline Occasionally & $10(11 \%)$ & $1(17 \%)$ & $1(3.7 \%)$ & $7(16.3 \%)$ & 0 & 0 \\
\hline
\end{tabular}

INSPEAR = International Network for the Study and Prevention of Emerging Antimicrobial Resistance.

43) in Western Europe. The routine use of gloves during the care of MRSA-infected patients ranged from $17 \%$ ( 1 of 6) in Africa to $100 \%$ (5 of 5 ) in the United States. The routine use of gowns ranged from $0 \%(0$ of 6$)$ in Africa to $65 \%$ (28 of 43) in Western Europe. The proportion of healthcare facilities in which hand washing was routinely performed in the patient's room ranged from $17 \%$ ( 1 of 6 ) in Africa to $62.8 \%$ (27 of 43) in Western Europe and 67\% (4 of 6) in South America. The proportion of healthcare facilities posting an isolation sign on the patient's door ranged from $0 \%(0$ of 6$)$ in African healthcare facilities to $67 \%$ ( 4 of 6) in South American healthcare facilities. Special procedures, such as a letter informing that the transferred patient is colonized or infected, were used by $40(44.4 \%)$ of the healthcare facilities, most in Western Europe (31 of $43,72 \%)$. Only $14(15.6 \%)$ of the healthcare facilities were systematically cohorting patients infected or colonized with MRSA, whereas 17 (18.9\%) did it occasionally (Table 3).

The implementation of a MRSA control program increased from $2.2 \%$ ( 2 of 90 ) of the healthcare facilities in 1985 to $10 \%$ (9 of 90) in 1990, 37.8\% (34 of 90) in 1995, and $61.1 \%$ (55 of 90$)$ in 1997.

We classified a healthcare facility as "high incidence" if its MRSA infection rate per patient-days was 0.4 or greater, which was the median infection rate in 1997 , and as "low incidence" if the rate was less than 0.4 per 1,000 patient-days. On univariate analysis, the only risk factor for high incidence was the mean number of beds per infection control nurse. Healthcare facilities with high incidence rates had a mean of 834 beds (standard deviation, 805) per infection control nurse, whereas healthcare facili- ties with low incidence rates had a mean of 318 beds (standard deviation, 209) per infection control nurse $(P=.02)$.

\section{Program to Control the Use of Antimicrobials}

Overall, the use of antimicrobials was controlled at 49 (54.4\%) of the healthcare facilities: $3(75 \%)$ of 4 in the United States, $25(59.5 \%)$ of 42 in Western Europe, 13 $(52 \%)$ of 25 in Eastern Europe, $3(50 \%)$ of 6 in South America, and $1(17 \%)$ of 6 in Africa. Formulary restriction was the most common control method used (40 of 49 , $81.6 \%$ ), followed by infectious disease or microbiology consultation (32 of $49,65.3 \%$ ) or stop orders (10 of 49 , $20.4 \%)$.

\section{Secular Trends in MRSA Infections}

Twenty-two healthcare facilities (Asia, $\mathrm{N}=1$; Western Europe, $\mathrm{N}=15$; Eastern Europe, $\mathrm{N}=2$; South America, $\mathrm{N}=3$; and United States, $\mathrm{N}=1$ ) provided data on the incidence per patient-days of hospitalization for 2 or more consecutive years, and 20 of them (91\%) had a quality assurance program (internal, 18 [81.8\%]; external, 14 $[63.6 \%])$. From the date of initiation of the surveillance program to the end of 1997, the incidence of MRSA infection per patient-days decreased in 9 healthcare facilities (41\%) and increased in 13 healthcare facilities (59\%). During this time, changes in MRSA infection rates ranged from $-54 \%$ to $+414 \%$.

\section{DISCUSSION}

The emergence of multidrug-resistant pathogens represents a major impending public health problem for healthcare facilities worldwide, but few data are available 
about how healthcare facilities have prepared to face the challenge of controlling multidrug-resistant pathogens. In addition, the control of multidrug-resistant pathogens may be hampered in developed countries by the increasing complexity of the healthcare delivery system and by attempts to reduce the costs of health care. In contrast, healthcare facilities in underdeveloped countries may lack basic microbiology and infection control resources. Therefore, we performed this survey to assess how healthcare facilities worldwide identify, survey, and control multidrug-resistant pathogens.

MRSA was chosen as a target organism among the numerous multidrug-resistant pathogens because it is a true pathogen that has spread in healthcare facilities worldwide, it is becoming increasingly resistant to antimicrobials, including glycopeptides, and recent evidence suggests that MRSA also may be spreading within the community. $13,18-23$ In addition, the subject is controversial and there is an ongoing polemic in the medical literature. Whereas some authors state that MRSA should be controlled because these organisms may soon become resistant to all antimicrobials and that infection control measures are beneficial, other authors suggest that the benefits of controlling MRSA are unclear because it is practically impossible to apply control measures and because attempts at controlling MRSA in healthcare facilities have failed. ${ }^{24-28}$

We found several important deficiencies in the microbiology, epidemiology, and infection control areas. In microbiology, our survey revealed that some laboratories have problems detecting or identifying resistance because of inadequate methods, as exemplified by the report of the isolation of vancomycin-resistant or teicoplanin-resistant $S$. aureus. Such clinical organisms had never been reported, yet no attempts were made to confirm the resistance or to alert public health authorities about their potential existence. This suggests that early warning systems may not exist in many countries and that laboratorians ignore or have no access to recommendations on how to detect emerging antimicrobial resistance and do not know to whom and when to send problematic isolates for confirmation or report the isolation of such organisms. In addition, potentially serious mistakes may be caused by the multiplicity of breakpoints when, as shown in this study, laboratories supposedly following one set of recommendations were using a disk designed for breakpoints recommended by another committee that contained an amount of antimicrobial different from the required amount. Such mistakes could be prevented if the national committees involved in antimicrobial susceptibility testing could reach a consensus on methods and breakpoints. Fortunately, several laboratories used a combination of different methods to determine susceptibility to oxacillin, which could decrease the risk of errors. If we are to identify emerging multidrug-resistant pathogens, it will be necessary to educate laboratorians on appropriate laboratory methods to use.

The fact that glycopeptide-intermediate $S$. aureus would not be detected by the laboratories using only the disk-diffusion method suggests that such strains could emerge in many areas of the world, remain undetected, and become endemic or transmitted to other patients and healthcare facilities both within and outside of a country.

The assessment of the surveillance methods used by INSPEAR healthcare facilities showed that the implementation of MRSA surveillance programs was generally late during the MRSA epidemic, as few healthcare facilities conducted such activities before 1990. The long delay in implementing surveillance programs while MRSA infection rates increased has led to the endemicity of patients infected or colonized with MRSA in many healthcare facilities worldwide. In addition, most healthcare facilities calculated only the proportion of all $S$. aureus that were methicillin resistant, a method that may poorly reflect the impact of infection control efforts. ${ }^{29}$ Fewer healthcare facilities used other denominators, such as the number of admissions, which is better for rate calculation, or the number of days of hospitalization, which is particularly useful for inter-healthcare facility or intra-healthcare facility comparison. These data show that MRSA continues to increase in incidence in many healthcare facilities worldwide and will be difficult to control without sustained infection control efforts. Furthermore, unless the types of rates or incidence data collected are changed, the measurement of secular trends or inter-hospital or intrahospital MRSA comparisons to evaluate the efficacy of control measures will be impossible.

MRSA infection rates for 1997 showed wide variations. Of interest, the same variations were observed within each category of healthcare facility when they were classified according to their number of beds or within each continent, even when the median rates of infection were similar. Therefore, the MRSA infection rates appear to be independent of the number of beds present in the healthcare facility or of the level of development of the country but dependent on the presence of an adequate number of trained infection control nurses, as shown in the analysis of risk factors for a high incidence rate of MRSA infection. This analysis showed that the infection control nurse in a healthcare facility with a low incidence rate was responsible for half the number of beds compared with the infection control nurse in a healthcare facility with a high incidence rate. Thus, additional infection control nurses and infection control support will be needed if we are to control MRSA and other multidrug-resistant pathogens.

The assessment of secular trends in MRSA infection clearly demonstrates that MRSA infections are far from being controlled in INSPEAR healthcare facilities. The incidence of MRSA infection increased in 59\% of the healthcare facilities from the date of initiation of the surveillance program to the end of 1997.

The presence of a limited number of infection control nurses in many healthcare facilities and the evaluation of the infection control precautions used by INSPEAR healthcare facilities may explain why MRSA infections are 
so difficult to control. The assessment of the use of isolation precautions recommended by the Centers for Disease Control and Prevention or considered as accepted practices revealed the existence of wide variations in the implementation of those precautions, even in developed countries. ${ }^{30}$ This was an assessment of policy. However, observational studies of infection control precautions nearly always reveal that practice is much lower than policy. The implementation of isolation precautions requires both the knowledge of the measures to use and the allocation of adequate resources; the poor compliance reported probably results from a combination of inadequate knowledge and resources. The poor familiarity with the recommended isolation precautions is demonstrated by the low rate of use of such inexpensive measures as posting an isolation sign on the patient's door. In contrast, the poor compliance with hospitalization in a private room, the use of gloves or gowns, and appropriate hand hygiene in the patient's room may be caused by inadequate education about recommended precautions but more likely reflects a lack of isolation facilities or infection control resources, even in developed countries.

Our study has several limitations. The survey was not performed in a representative sample of hospitals, the quality of the data collected could not always be assessed, and variations in culturing practices could have an impact on the data. However, our results strongly suggest that the 90 INSPEAR healthcare facilities located in 30 countries with different levels of resources are not optimally prepared to control MRSA and other multidrug-resistant pathogens. Thus, the emergence of vancomycin-resistant $S$ aureus or any other pathogen resistant to all antimicrobials could lead to a public health disaster. The most effective way to control and prevent the emergence of MRSA or other multidrug-resistant pathogens is to make clinical microbiology laboratories and the medical community aware of and prepared for potential problems. Preparedness implies that laboratories have the capacity to detect emerging resistance, difficult resistance phenotypes, or both; screen patients for colonization; share antimicrobial resistance data with infection control personnel and clinicians in a timely fashion; assess risk factors for emergence of resistance; monitor MRSA and multidrug-resistant pathogens with surveillance programs whose results are usable for control and prevention; and implement efficient isolation precautions (eg, private rooms with hand washing facilities and the allocation of gloves).

Allowing MRSA to become endemic at many healthcare facilities worldwide raises an important infection control challenge. Do we attempt to control MRSA now before vancomycin resistance becomes prevalent or do we wait until MRSA has become vancomycin resistant and we have to deal with a worldwide catastrophe? Data show that aggressive screening and isolation of vancomycinresistant enterococci or MRSA can control or eliminate such multidrug-resistant pathogens. ${ }^{24,25}$ Although these measures are cost-effective, they are expensive and have been difficult to fund because their implementation requires the training of microbiology and infection control personnel and the strengthening of microbiology, epidemiology, and infection control capacities of healthcare facilities worldwide. Funding of these activities should become a priority at the local, national, and international levels before multidrug-resistant pathogens that are virtually untreatable emerge, are transmitted nationally and internationally, become endemic in our healthcare facilities, and are transmitted to the community.

\section{REFERENCES}

1. Go ES, Urban C, Burns J, et al. Clinical and molecular epidemiology of Acinetobacter infections sensitive only to polymyxin B and sulbactam. Lancet 1994;344:1329-1332.

2. Richard P, Le Floch R, Chamoux C, Pannier M, Espaze E, Richet H. Pseudomonas aeruginosa outbreak in a burn unit: role of antimicrobials in the emergence of multiply resistant strains. I Infect Dis 1994; 170:377-383.

3. French GL, Shannon KP, Simmons N. Hospital outbreak of Klebsiella pneumoniae resistant to broad-spectrum cephalosporins and $\beta$-lactam$\beta$-lactamase inhibitor combinations by hyperproduction of SHV5 $\beta$-lactamase. J Clin Microbiol 1996;34:358-363.

4. Neuwirth C, Siebor E, Pechinot A, Kazmierczak A. Outbreak of TEM24-producing Enterobacter aerogenes in an intensive care unit and dissemination of the extended spectrum $\beta$-lactamase to other members of the family enterobacteriaceae. J Clin Microbiol 1996;34:76-79.

5. Morosini MI, Canton R, Martinez-Beltran J, et al. New extended-spectrum TEM-type $\beta$-lactamase from Salmonella enterica isolated in a nosocomial outbreak. Antimicrob Agents Chemother 1995;39:458-461.

6. Pagani L, Luzzaro F, Ronza P, et al. Outbreak of extended spectrum $\beta$ lactamase producing Serratia marcescens in an intensive care unit. FEMS Immunol Med Microbiol 1994;10:39-46.

7. Johnson DR, Love-Dixon MA, Brown WJ, Levine DP, Downes FP, Hall WN. Delayed detection of an increase in resistant Acinetobacter baumannii at a Detroit hospital. Infect Control Hosp Epidemiol 1992;13:394398.

8. Meyer KS, Urban C, Eagan JA, Berger BJ, Rahal JJ. Nosocomial outbreak of Klebsiella infection resistant to late-generation cephalosporins. Ann Intern Med 1993;119:353-358.

9. Trick WE, Kuehnert MJ, Quirk SB, et al. Regional dissemination of vancomycin-resistant enterococci resulting from interfacility transfer of colonized patients. J Infect Dis 1999:180:391-396.

10. Austin DJ, Anderson RM. Transmission dynamics of epidemic methicillin-resistant Staphylococcus aureus and vancomycin-resistant enterococci in England and Wales. J Infect Dis 1999;179:883-891.

11. Kim WJ, Weinstein RA, Hayden MJ. The changing molecular epidemiology and establishment of endemicity of vancomycin resistance in enterococci at one hospital over a 6-year period. I Infect Dis 1999;179:163-171.

12. Michel M, Gutmann L. Methicillin-resistant Staphylococcus aureus and vancomycin-resistant enterococci: therapeutic realities and possibilities. Lancet 1997;349:1901-1906.

13. Smith TL, Pearson ML, Wilcox KR, et al. Emergence of vancomycin resistance in Staphylococcus aureus. Glycopeptide-intermediate Staphylococcus aureus working group. N Engl J Med 1999;340:493-501.

14. Barber M. Methicillin-resistant staphylococci. I Clin Pathol 1961;14:385-393.

15. Chabbert YA, Baudens JG. Souches de staphylocoques resistants naturellement a la meticilline et a la 5 methyl-3-phenyl-4-iso-oxazyl penicilline (P12). Annales de l'Institut Pasteur (Paris) 1962;103:222230.

16. Richet $H$, Wiesel M, Le Gallou F, Andre-Richet B, Espaze E. Methicillin-resistant Staphylococcus aureus control in hospitals: the French experience. Infect Control Hosp Epidemiol 1996;17:509-511.

17. Farrington M, Redpath $C$, Trundle C, Coomber S, Brown NM. Winning the battle but losing the war: methicillin-resistant Staphylococcus aureus (MRSA) infections at a teaching hospital. QJM 1998;91:539-548.

18. Hiramatsu K, Aritaka N, Hanaki H, et al. Dissemination in Japanese hospitals of strains of Staphylococcus aureus heterogeneously resistant to vancomycin. Lancet 1997;350:1670-1673.

19. Ploy MC, Grelaud C, Martin C, de Lumley L, Denis F. First clinical isolate of vancomycin-intermediate Staphylococcus aureus in a French Hospital. Lancet 1998;351:1212. 
20. Maguire GP, Arthur AD, Boustead PJ, Dwyer B, Currie BJ. Clinical experience and outcomes of community-acquired and nosocomial methicillin-resistant Staphylococcus aureus in a northern Australian hospital. J Hosp Infect 1998;38:273-281.

21. Four pediatric deaths from community-acquired methicillin-resistant Staphylococcus aureus: Minnesota and North Dakota, 1997-1999. MMWR 1999;48:707-710.

22. L'Heriteau F, Lucet JC, Scanvic A, Bouvet E. Community-acquired methicillin-resistant Staphylococcus aureus and familial transmission. JAMA 1999;282:1038-1039.

23. Shahin R, Johnson IL, Jamieson F, McGeer A, Tolkin J, Ford-Jones EL. Methicillin-resistant Staphylococcus aureus carriage in a child care center following a case of disease: Toronto Child Care Study Group. Arch Pediatr Adolesc Med 1999;153:864-868.

24. Chaix C, Durand-Zaleski I, Alberti C, Brun-Buisson C. Control of endemic methicillin-resistant Staphylococcus aureus: a cost-benefit analysis in an intensive care unit. JAMA 1999;282:1745-1751.

25. Schentag JJ, Hyatt JM, Carr JR, et al. Genesis of methicillin-resistant Staphylococcus aureus (MRSA): how treatment of MRSA infections has selected for vancomycin-resistant Enterococcus faecium and the importance of antibiotic management and infection control. Clin Infect Dis 1998;26:1204-1214.

26. Jernigan JA, Titus MG, Groschel DH, Getchell-White S, Farr BM. Effectiveness of contact isolation during a hospital outbreak of methicillin-resistant Staphylococcus aureus. Am J Epidemiol 1996;143:496504.

27. Barrett SP, Mummery RV, Chattopadhyay B. Trying to control MRSA causes more problems than it solves. J Hosp Infect 1998:39:85-93.

28. Fazal BA, Telzak EE, Blum S, Turett GS, Ptersen-Fitzpatrick FE, Lorian V. Trends in the prevalence of methicillin-resistant Staphylococcus aureus associated with discontinuation of an isolation policy. Infect Control Hosp Epidemiol 1996;17:372-374.

29. Harbarth S, Martin Y, Rohner P, Henry N, Auckenthaler R, Pittet D. Effect of delayed infection control measures on a hospital outbreak of methicillin-resistant Staphylococcus aureus. J Hosp Infect 2000;46:43-49.

30. Garner IS. Guideline for isolation precautions in hospitals: the Hospital Infection Control Practices Advisory Committee. Infect Control Hosp Epidemiol 1996;17:53-80. 